\title{
Immunolocalization of Poly ADP-Ribose on Drosophila Polytene Chromosomes
}

\section{Anna Sala and Davide F.V. Corona}

\begin{abstract}
Poly ADP-ribosylation (PARylation) is a posttranslational protein modification catalyzed by poly ADP-ribose polymerases (PARPs). Poly ADP-ribose metabolism is involved in a wide range of biological processes, such as maintenance of genome stability, transcriptional regulation, energy metabolism, and programed cell death. Recently, chromatin components, including histones, have been shown to be targets of PARylation. Unlike mammals, which have several PARP-encoded genes, the model organism Drosophila melanogaster has only one PARP gene, highly related to mammalian PARPl. These features make flies a great model system to study PARP biology. Commercially available antibodies recognizing this covalent modification have made possible the development of immunofluorescence approaches to study PARylation of chromatin components. Here, we present a protocol to immunostain polytene chromosomes of the model system D. melanogaster.
\end{abstract}

Key words: PARP, Poly ADP-Ribose, Drosophila melanogaster, Polytene chromosomes

\section{Introduction}

Poly ADP-ribosylation (PARylation) is a covalent modification of proteins catalyzed by poly ADP-ribose polymerases (PARPs), a family of abundant and ubiquitous nuclear enzymes that transfer and polymerize $\mathrm{ADP}$-ribose units from $\mathrm{NAD}^{+}$on a variety of nuclear proteins to form a branched polymer known as poly ADPribose (PAR) $(1,2)$. PAR metabolism is implicated in a wide range of biological processes, such as maintenance of genomic stability, transcriptional regulation, energy metabolism, cell death, and also mechanisms suppressing carcinogenesis $(3-5)$. By now, a growing body of evidences suggests that PARP physically and functionally interacts also with chromatin-modifying enzymes, indicating that PARylation may play an important epigenetic role in combination 
with other covalent or ATP-dependent chromatin modifications $(3,5,6)$. Therefore, the identification of PAR on chromatin could be a useful tool to study the epigenetic role of PARP. In Drosophila melanogaster, some specialized cells undergo repeated rounds of DNA replication without cell division forming a giant polytene chromosome. A useful feature of these cells is that they are in interphase and that their chromosomes have a pattern of dark and light bands (bands and interbands, respectively) that like a bar code is unique for each chromosome. The banding pattern of Drosophila polytene chromosomes provides an excellent visualization of transcriptionally active chromatin sites and important information about their chromatin structure $(7,8)$. The commercial availability of antibodies recognizing PAR has recently allowed the detection of this epigenetic marker in eukaryotic chromatin. Here, we describe a method to localize PAR on Drosophila polytene chromosomes by immunofluorescence.

\section{Materials}

\subsection{Drosophila Stock Keeping}

2.2. Extraction of Salivary Glands and Squashes

2.3. Trichloroacetic Acid Treatment
1. K12 medium (USBiological).

2. Yeast instant success (ESKA SNC).

1. Precision Stainless Steel Dissection Tweezers No. 5.

2. Dissection solution (DS): $0.7 \%(\mathrm{w} / \mathrm{v}) \mathrm{NaCl}$.

3. Petri dish.

4. $3.7 \%$ formaldehyde stock solution: Weigh $0.37 \mathrm{~g}$ of paraformaldehyde (SIGMA) and dissolve in a $10 \mathrm{ml}$ final volume of distilled $\mathrm{H}_{2} \mathrm{O}$. Store in aliquots of $100 \mu \mathrm{l}$ at $-20^{\circ} \mathrm{C}$. This solution is stable for several months.

5. Fixing solution: $45 \%(\mathrm{v} / \mathrm{v})$ acetic acid, $1.8 \%(\mathrm{w} / \mathrm{v})$ formaldehyde. Prepare by adding $100 \mu \mathrm{l}$ of $3.7 \%$ formaldehyde stock solution to $100 \mu \mathrm{l}$ of glacial acetic acid. Prepare fresh for every experiment.

6. Micro Cover Glass $22 \times 22$ Cover slip (PRESTIGE).

7. Super Frost $26 \times 76 \times 1 \mathrm{~mm}$ Slides (Thermo Fisher).

8. Blotting paper.

9. Diamond tip pen.

10. Liquid nitrogen.

1. Razor blade.

2. Glass Pasteur.

3. $50 \%(\mathrm{w} / \mathrm{v})$ trichloroacetic acid (TCA) stock solution: Weigh $50 \mathrm{~g}$ of TCA (SIGMA) and dissolve in a $100 \mathrm{ml}$ final volume of 
distilled $\mathrm{H}_{2} \mathrm{O}$. Store at $4^{\circ} \mathrm{C}$. This solution is light sensitive, cover it with aluminum foil to make it stable for several months.

4. $10 \%$ TCA: Add $10 \mathrm{ml}$ of $50 \%$ TCA stock solution to $40 \mathrm{ml}$ of distilled $\mathrm{H}_{2} \mathrm{O}$. Store at $4^{\circ} \mathrm{C}$. This solution is light sensitive, cover it with aluminum foil to make it stable for several months.

5. Absolute ethanol.

6. $70 \%$ ethanol: Add $70 \mathrm{ml}$ of absolute ethanol to $30 \mathrm{ml}$ of distilled $\mathrm{H}_{2} \mathrm{O}$ and invert to mix.

7. $90 \%$ ethanol: Add $90 \mathrm{ml}$ of absolute ethanol to $10 \mathrm{ml}$ of distilled $\mathrm{H}_{2} \mathrm{O}$ and invert to mix.

\subsection{Immunostaining}

1. Slide racks.

2. $2 \%(\mathrm{w} / \mathrm{v})$ NaAzide stock solution: Weigh $2 \mathrm{~g}$ of NaAzide and dissolve in a $100 \mathrm{ml}$ final volume of distilled $\mathrm{H}_{2} \mathrm{O}$. Store at room temperature.

3. Blocking solution: $3 \%$ bovine serum albumin (BSA), $0.1 \%$ Triton X-100 in PBS $1 \times(\mathrm{pH} 7.4)$. To make 11 , dissolve $30 \mathrm{~g}$ of BSA powder in $100 \mathrm{ml}$ of PBS $10 \times(\mathrm{pH} 7.4), 10 \mathrm{ml}$ of $10 \%$ Triton X-100, and $890 \mathrm{ml}$ of distilled $\mathrm{H}_{2} \mathrm{O}$ with the help of a magnetic stirrer. Make the solution $0.02 \%$ NaAzide to store it for several weeks at $4^{\circ} \mathrm{C}$.

4. PBS-T: $0.1 \%$ Triton $\mathrm{X}-100$ in PBS $1 \times(\mathrm{pH} 7.4)$. To make 11 , add $10 \mathrm{ml}$ of $10 \%$ Triton X-100 to $100 \mathrm{ml}$ of PBS $10 \times(\mathrm{pH}$ 7.4) and $890 \mathrm{ml}$ of distilled $\mathrm{H}_{2} \mathrm{O}$, and mix by inverting.

5. Monoclonal antibody against PAR (clone 10H; ALEXIS).

6. Secondary antibody: Goat anti-mouse IgG-FITC (Santa Cruz Biotechnology).

7. Vectashield Mounting Medium with DAPI (Vector Laboratories).

\section{Methods}

Polytene chromosomes are present in many larval tissues in Drosophila. They can easily be prepared from salivary glands of third instar larvae, where the degree of polytenization is maximal. These giant chromosomes represent an excellent material to localize proteins of interest or epigenetic markers, such as PAR, on chromatin by cytological methods, for example by immunofluorescence. While the addition of PAR moieties is catalyzed by members of the PARP family, the removal of PAR chains from the modified proteins is catalyzed by the poly ADP-ribose glycohydrolase enzyme (PARG), which is the primary enzyme responsible for PAR turnover in vivo (9). Since PARylation is a reversible posttranslational 


\subsection{Immuno-} fluorescence

3.1.1. Growth and Collection of Third Instar Larvae
3.1.2. Extraction of Salivary Glands and Chromosome Squashes protein modification, classical immunostaining protocols (10) had to be modified and adapted in order to detect PAR on polytene chromosomes. To obtain reliable and reproducible results, it is important to be sure to completely block PARG enzymatic activity and rapidly process the slides. In order to prevent catabolism of the PAR polymers by PARG, samples must be first fixed in TCA in addition to formaldehyde (see Fig. la). This immunostaining protocol allows the detection of quantitative differences in the relative amount of PAR loaded on polytene chromosomes. Indeed, while PAR chromatin levels in Parp mutants are reduced when compared with those of wild-type polytene chromosomes (see Fig. 1b), Parg mutant chromatin displays an increase in PAR staining when compared to wild-type chromatin (see Fig. lb). It is also important to note that this immunostaining protocol for PAR detection is compatible with double staining.

1. Place about 15 males and 15 females wild-type flies in vials containing a nutrient rich medium (i.e., Kl2). To improve growth and egg deposition, add few granules of dried yeast on top of the medium.

2. Let flies lay eggs at $18^{\circ} \mathrm{C}$ for $48 \mathrm{~h}$, this ensures larval growth under not crowded conditions, and allows larvae to develop till third instar stage (approximately 8-10 days after egg laying).

3. Grow larvae at $18^{\circ} \mathrm{C}$ to have large salivary gland cells, with a high level of polytenization, thus obtaining a better polytene chromosomes quality.

4. With a pair of tweezers pick crawling third instar larvae along the vial walls. Do not take larvae that are dead or have just started to pupate.

1. Transfer larvae from the vial to a drop of DS. Wash larvae by rinsing them three times in DS.

2. Dissect one larva at a time in a $50 \mu \mathrm{l}$ drop of DS in a Petri dish. To obtain best results, clamp the middle of the larval body with one tweezers and pull the mouth hook outside the body with another tweezers. Glands will come attached to the anterior parts of the larval tissues. Remove most of the fat bodies without separating the two glands, thus facilitating the following steps.

3. With the help of the tweezers, transfer the glands directly to $20 \mu \mathrm{l}$ of fixing solution on a coverslip.

4. Fix glands for $2 \mathrm{~min}$ at room temperature.

5. Put a slide on the coverslip and quickly flip in order to bring the slide down and the coverslip up. Glands are visible as an opaque halo. Tap gently the coverslip with the tip of a pencil for about 10-20 s until cells are broken and chromosomes spread on the slide (see Note 1 ). 
a

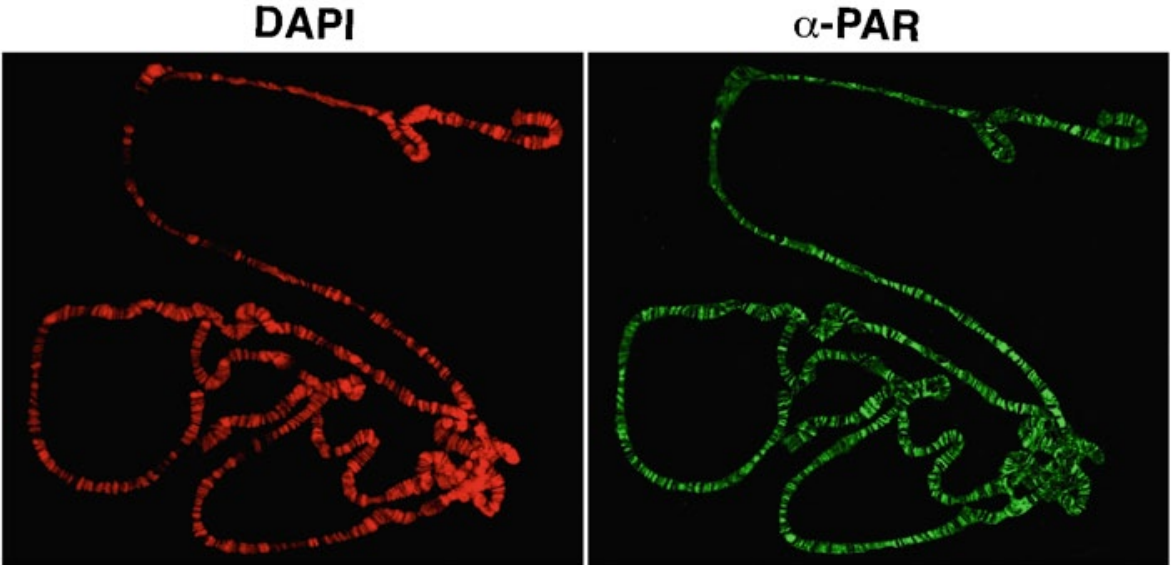

b

wt

Parp

Parg

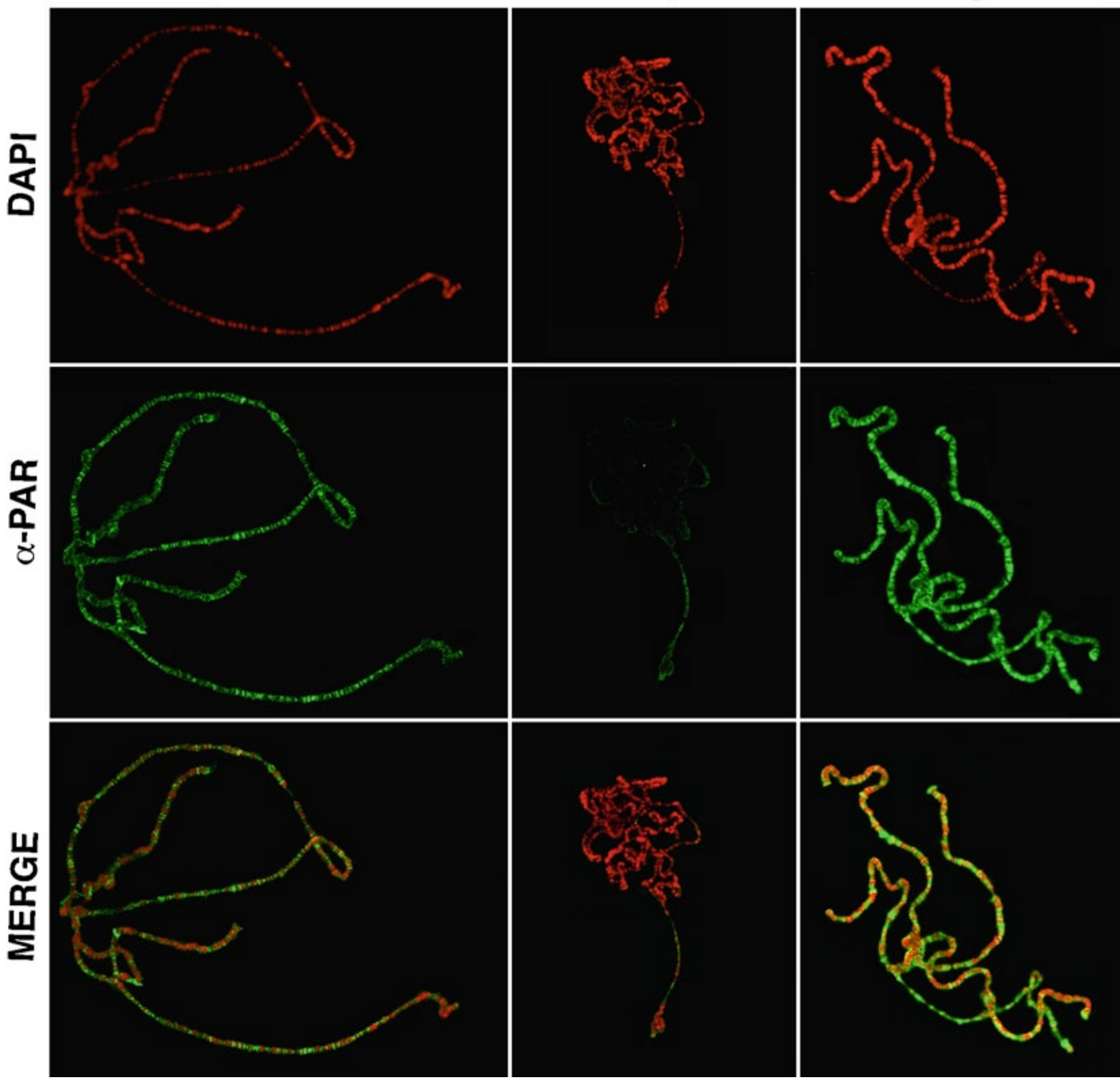

Fig. 1. PAR localization on polytene chromosomes. (a) Distribution of PAR (green) and DAPI (red) on polytene chromosomes from wild-type third instar larvae. (b) Immunostaining of PAR (green) on polytene chromosomes from wild-type (wt), homozygous Parp ${ }^{\text {C03256 }}$ (Parp) (11), and Parg ${ }^{27.1}$ (Parg) mutants (12) third instar larvae. Chromosomes were also stained with DAPI to visualize DNA $(r e d)$. All images were captured with the same camera settings used for the wild-type control. This analysis revealed a reduction of the PAR immunostaining signal on Parp mutant chromosomes and an increase of PAR staining on Parg mutant chromosomes. 
3.1.3. TCA Treatment

3.1.4. Immunostaining and Detection
6. Turn the slide with the coverslip down to the blotting paper and spread chromosomes with one finger. Check the spread very quickly under a phase contrast microscope. If chromosomes are not spread enough, repeat spreading as mentioned above (see Note 2 ).

7. Mark positions of the squashed area and coverslip borders on the back of the slide using a diamond tip pen.

8. Place slides in liquid nitrogen.

1. Take off slides from liquid nitrogen and quickly remove coverslip with a razor blade.

2. Put slides on blotting paper with squashed area up and drop frozen $\left(-20^{\circ} \mathrm{C}\right)$ absolute ethanol with a glass Pasteur directly on chromosomes and incubate $1 \mathrm{~min}$ at room temperature. Remove ethanol letting it drop on blotting paper.

3. Rapidly put on slides cold $\left(4^{\circ} \mathrm{C}\right) 10 \%$ TCA, using a glass Pasteur and incubate $10 \mathrm{~min}$ at room temperature (see Note 3 ). Remove all TCA letting it drop on blotting paper.

4. Dehydrate slides by washing in $70 \%$ ethanol, $90 \%$ ethanol, and absolute ethanol $1 \mathrm{~min}$ each at room temperature. For each wash, drop directly ethanol on squashed area with a glass Pasteur.

5. Drain the last absolute ethanol wash on the blotting paper and air-dry slides.

1. Transfer slides in a rack full of blocking solution and incubate for $\mathrm{l} \mathrm{h}$ at room temperature.

2. Discard blocking solution and wash slides three times for 10 min each with PBS-T.

3. Prepare primary antibody solution diluting 1:30 Anti-PAR (clone $10 \mathrm{H}$ ) in blocking solution.

4. Put $15 \mu$ of primary antibody dilution on a coverslip and lay over the slide avoiding bubbles formation. Incubate overnight at $4^{\circ} \mathrm{C}$ in a humid chamber.

5. After the antibody overnight incubation, remove the coverslip by rinsing the slides in PBS-T.

6. Wash three times in PBS-T for $10 \mathrm{~min}$ at room temperature (see Note 4).

7. Prepare secondary antibody solution diluting $1: 50$ anti-mouse IgG-FITC conjugated in blocking solution (see Note 5 ).

8. Put $15 \mu \mathrm{l}$ of secondary antibody dilution on a coverslip and lay over the slide avoiding bubbles formation. Incubate $90 \mathrm{~min}$ at room temperature in a humid chamber in the dark.

9. Remove the coverslip by rinsing the slides in PBS-T and wash three times in PBS-T for $10 \mathrm{~min}$ at room temperature (see Note 6). 
3.2. Cytological Analysis of Immunostained Slides

3.3. Analysis and Assembly of Immunostained Pictures
10. Mount slides putting $15 \mu \mathrm{l}$ of DAPI-containing mounting media on a coverslip and lay over the slide avoiding bubbles formation.

1. Examine the slides under a microscope (see Note 7).

2. Acquire images of intact and widespread chromosomes using a DAPI and an FITC filter. We used a Leica DM IRB microscope equipped with a DC300F camera. The images were acquired using the QFluor (Leica) software.

3. Store the images files in "TIFF" format and in "grayscale" color mode for subsequent images processing.

We assembled and elaborated images using Adobe PhotoShop software. The following instructions are examples of how to assemble the acquired pictures.

1. Select the best chromosomes for image analysis.

2. Usually, for every chromosome there are two or three images (for single or double staining, respectively): the DAPI staining and the immunostaining pictures. These images are 8-bit "Grayscale" color mode TIFF format files.

3. Open all the files in Adobe PhotoShop.

4. Convert the images from "Grayscale" to "RGB" color mode. Then, "Copy" the DAPI image and open a new document. The resulting document has a layer with three channels: red, green, and blue. Save this file as a new, psd file making sure to have a black background.

5. Create a pseudocolor pictures for each channel, by pasting the DAPI image in the blue channel, the FITC staining in the green channel and eventually the rhodamine staining in the red.

6. To reduce the background and improve the signal to noise, adjust the levels of each channel.

7. Visualize each channel by selecting or unselecting each of them. For a view of the overlay image, select the RGB channel.

\section{Notes}

1. Start tapping from the middle of the area containing glands and continue roundly to the border of the coverslip. Tapping should be performed using the right pressure, which requires adjustment in different experiments.

2. Good preparations should contain well-spread chromosomes with an evident banding and not fragmented. Wrapped chromosomes in unbroken nuclei depend on insufficient tapping 
while high level of fragmentation indicates an excessive pressure during tapping or that coverslip was moved during squash.

3. This is really the critical step of the whole PAR immunodetection experiment. A discontinuous staining in the slide could depend on a short incubation (less than $10 \mathrm{~min}$ ) with TCA. Shorter time incubations could also affect the reproducibility of the staining in different experiments.

4. If you want to conduct a double staining using another primary antibody, raised in a host different from mouse, at this point continue as in steps 3-6 (in Subheading 3.1.4). Primary antibody dilutions have to be adjusted using serial dilutions.

5. It is extremely important to use secondary antibodies solutions containing less than $30 \%$ glycerol as glycerol may interfere with PAR staining.

6. If you are conducting a double staining, prepare secondary antibody solution, for the second primary antibody, diluting 1:100 IgG-Cy3 conjugated in blocking solution and repeat steps 8-10 (in Subheading 3.1.4).

7. For best results, examine slides as soon as they are ready. Storing slides at $4^{\circ} \mathrm{C}$ may drop the quality of PAR staining.

\section{Acknowledgments}

We would like to thank Antonia Maria Rita Ingrassia for her useful technical support in the writing of the materials section. We also would like to thank the members of the lab for their critical reading and feedbacks on this manuscript. A.S. was supported by an FIRC fellowship. This work was supported by grants from Fondazione Telethon, Giovanni Armenise Harvard Foundation, FIRB-MIUR, AIRC, and Compagnia San Paolo to D.F.V.C.

\section{References}

1. D'Amours D, Desnoyers S, D'Silva I, Poirier GG (1999) Poly(ADP-ribosyl)ation reactions in the regulation of nuclear functions. Biochem J 342(Pt 2):249-268

2. Schreiber V, Dantzer F, Ame JC, de Murcia G (2006) Poly(ADP-ribose): novel functions for an old molecule. Nat Rev Mol Cell Biol 7:517-528

3. Hassa PO, Hottiger MO (2008) The diverse biological roles of mammalian PARPS, a small but powerful family of poly-ADP-ribose polymerases. Front Biosci 13:3046-3082
4. Miwa M, Masutani M (2007) PolyADPribosylation and cancer. Cancer Sci 98: 1528-1535

5. Quenet D, El Ramy R, Schreiber V, Dantzer F (2009) The role of poly(ADP-ribosyl)ation in epigenetic events. Int J Biochem Cell Biol 41:60-65

6. Sala A, La Rocca G, Burgio G, Kotova E, Di Gesu D, Collesano M, Ingrassia AM, Tulin AV, Corona DF (2008) The Nucleosome Remodeling ATPase ISWI is Regulated by poly-ADP-ribosylation. PLoS Biol 6:e252 
7. Skaer RJ (1977) Interband transcription in Drosophila. J Cell Sci 26:251-266

8. Spierer A, Spierer P (1984) Similar level of polyteny in bands and interbands of Drosophila giant chromosomes. Nature 307:176-178

9. Davidovic L, Vodenicharov $M$, Affar EB, Poirier GG (2001) Importance of poly(ADPribose) glycohydrolase in the control of poly(ADP-ribose) metabolism. Exp Cell Res 268:7-13

10. Corona DF, Armstrong JA, Tamkun JW (2004) Genetic and cytological analysis of Drosophila chromatin-remodeling factors. Methods Enzymol 377:70-85

11. Kotova E, Jarnik M, Tulin AV (2010) Uncoupling of the transactivation and transrepression functions of PARPl protein. Proc Natl Acad Sci USA 107: 6406-6411

12. Hanai S, Kanai M, Ohashi S, Okamoto K, Yamada M, Takahashi H, Miwa M (2004) Loss of poly(ADP-ribose) glycohydrolase causes progressive neurodegeneration in Drosophila melanogaster. Proc Natl Acad Sci USA 101: 82-86 